\title{
A Case of Clear Cell Sarcoma of Kidney in An Adult: An Extremely Rare Presentation
}

\author{
Pragya Nirav Vakani ${ }^{1 *}$, Mukesh Maheshwari ${ }^{1}$, Jainil Maheshwari ${ }^{2}$, Anshul Agarwal ${ }^{3}$, \\ Pragnesh Patel $^{3}$ and Chandresh Shah ${ }^{3}$ \\ 'Department of Pathology, Nirmaan Pathology Laboratory, Ahmedabad, Gujarat, India \\ ${ }^{2}$ Research Student, NHL Medical College, Ahmedabad, Gujarat, India \\ ${ }^{3}$ Department of Urology Deep Urosurgical Hospital, Ahmedabad, Gujarat, India
}

\begin{abstract}
Clear cell sarcoma of the kidney (CCSK) is a malignant renal tumor of childhood with a propensity to metastasize to bone and other organs. It is extremely rare in adults, must be differentiated from clear cell carcinoma of kidney which is more common in adults. We report a case of CCSK in a 45 year old female, confirmed by Immunohistochemistry (IHC)\& managed with radical nephrectomy. The aim of this case report is to increase the awareness about this entity in adults with brief review of relevant literature.
\end{abstract}

Keywords: Clear Cell Sarcoma, Immunohistochemistry, Kidney.

\section{Introduction}

Clear cell sarcoma of kidney is a rare tumor $(3-5 \%$ of childhood renal tumor), its mean age of diagnosis is 36 months with a range of 2 months -14 years ${ }^{[1]}$. It has propensity to metastasize to the bone \& has unfavorable prognosis. Reports of these tumor diagnosed in adults is rare ${ }^{[2-5]} \&$ its treatment is controversial with combination of surgery \& chemo radiotherapy. It is necessary to differentiate it from clear cell renal cell carcinoma which is most common in adults ${ }^{[2]}$. Immunohistochemistry is needed for confirmation of diagnosis, as this entity is not common in this age.

\section{Case Report}

A 47 year female presented with complaints of Left sided flank pain, fullness in abdomen \& hematuria since 1 month. USG \& CT scan with I.V. contrast revealed huge well defined heterogeneously enhancing mass lesion involving upper-mid poles of Left kidney with localized exophytic component with mass effect and without renal vein thrombosis. Radical nephrectomy was done under aseptic precaution in GA with Transabdominal approach. The patient was discharged post operative 5 days without complication.

Gross examination shows Left sided radical nephrectomy measuring $13 \times 8 \times 8 \mathrm{~cm}$ in size. Cut section shows greybrown solid-cystic mass at upper-mid pole of kidney measuring $8 \times 7 \times 6 \mathrm{~cm}$ in size. Extensive hemorrhage \& necrosis are seen [Figure 1].

Histo-pathological examination revealed diffuse monotonous proliferation of medium sized cells in nests separated by thin fibro vascular septa. The cells show fine chromatin with indistinct cell border \& clear cytoplasm [Figure 2]. Capsule, perinephric fat, fascia gerota, margin of vascular pedicle, renal sinus, ureter \& adrenal gland were free from the tumor.

On Immunohistochemistry tumor cell expressed positivity for vimentin, bcl-2 \& cyclin D1 which favors CCSK [Figure-3] and were immunonegative for Cytokeratin, EMA, Pax8 [Figure 4], Mic-2, Synaptophysin, Chromogranin A, Inhibin, Melan-A, CD-10, PgR, Desmin, SMA \& c-kit (to rule out clear cell carcinoma of kidney, rhabdoid tumor, neuroendocrine tumor, mesoblastic nephroma).

Finally on the basis of histomorphology and Immunohistochemistry diagnosis of clear cell sarcoma was given. Patient was referred to onco-physician and chemoradiotherapy was started.

\section{Discussion}

Clear cell sarcoma of kidney was first described in 1978 by three distinct groups: Beckwith and Palmer ${ }^{[6]}$, Morgan and Kidd ${ }^{[7]}$, and Marsden and coworkers ${ }^{[8]} \&$ it was initially named "bone metastasizing renal tumor of childhood" since it has predilection for skeletal metastasis. It is most frequently misdiagnosed renal tumor in children and it is rarely diagnosed in adults ${ }^{[2-3]}$. Males are slightly more affected (2:1).

Morphologically various patterns of clear cell sarcoma are noted with classical pattern of nests \& cords separated by thin fibro vascular septae which is focally present in every case. The other patterns are myxoid (50\%), sclerosing 


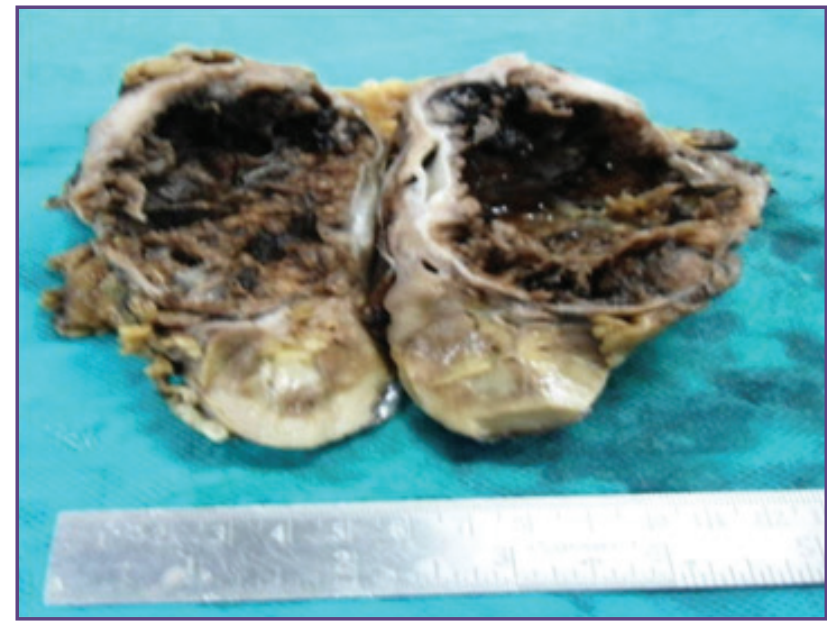

Fig. 1: Gross appearance of clear cell sarcoma of left kidney shows grey-brown solid-cystic mass at upper-mid pole of kidney measuring $8 \times 7 \times 6 \mathrm{~cm}$ in size with extensive hemorrhage \& necrosis.

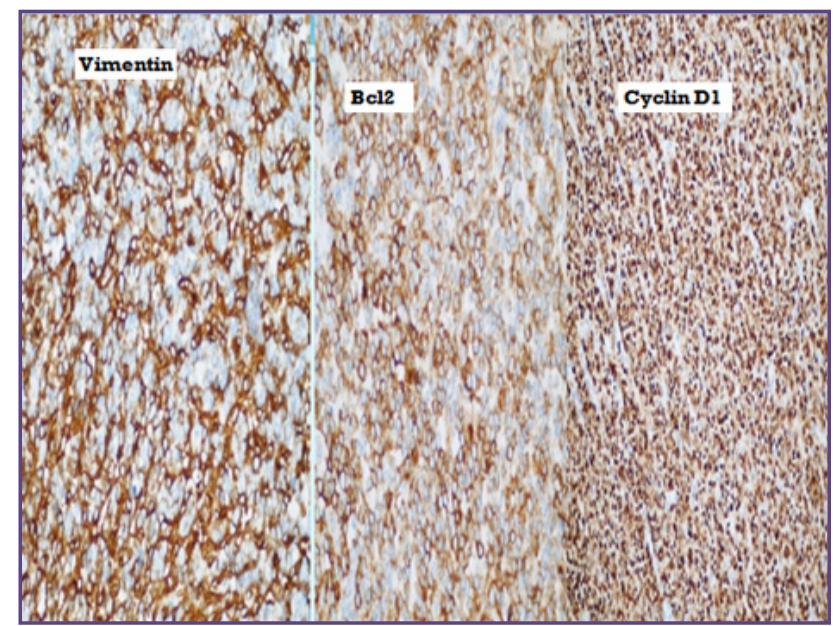

Fig. 3: (IHC stain- 20X) Clear cell sarcoma of kidneyvimentin, cyclin D1 \& BCl2 positive in tumor cells.

(35\%), cellular (26\%), epitheloid (13\%), palisading verocay body $(11 \%)$, spindle cell $(7 \%)$, storiform $(4 \%)$ and anaplastic $(2.6 \%)^{[1]}$. The nuclei of the CCSK are uniform with smooth chromatin and scant cytoplasm. On IHC tumor cells are consistently positive for vimentin \& cyclin D1 with CD34 positivity in thin walled capillaries ${ }^{[1,9]}$. Tumor cells are negative for Cytokeratin, EMA, WT1, CD99, Desmin and Synaptophysin.

Bone metastasis occurs in $17-40 \%$ of cases of CCSK tumor with the common site being of brain, regional lymph nodes, lungs, liver and soft tissue ${ }^{[10]}$.

Molecular genetics of CCSK is not fully elucidated. Internal tandem duplications in the BCOR gene (BCL6 co repressor) were identified in $100 \%(20 / 20)$ of CCSK of

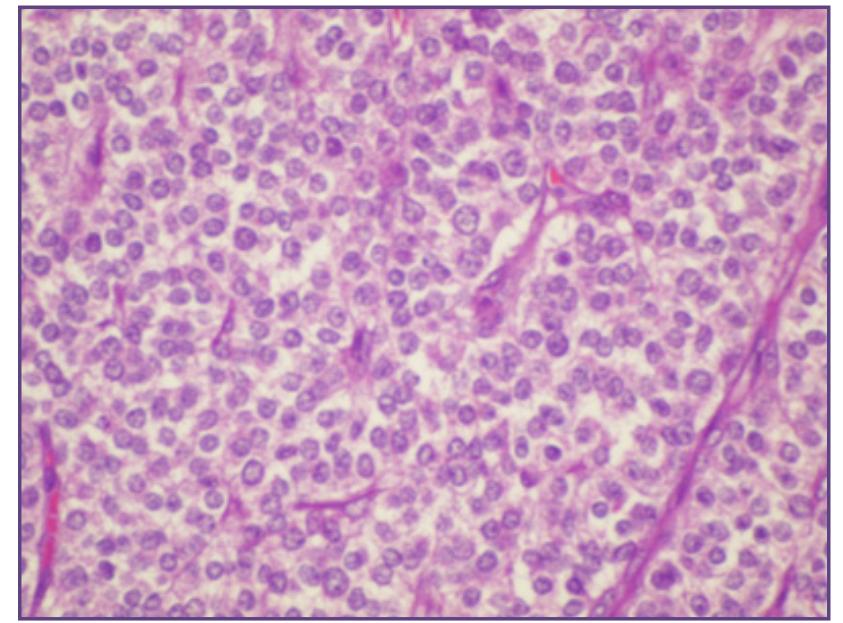

Fig. 2: H\&E stain 40x, Clear cell sarcoma of kidney. Tumor cells showing clear cytoplasm and centrally placed nuclei arranged in nesting pattern separated by fibro-vascular stroma.

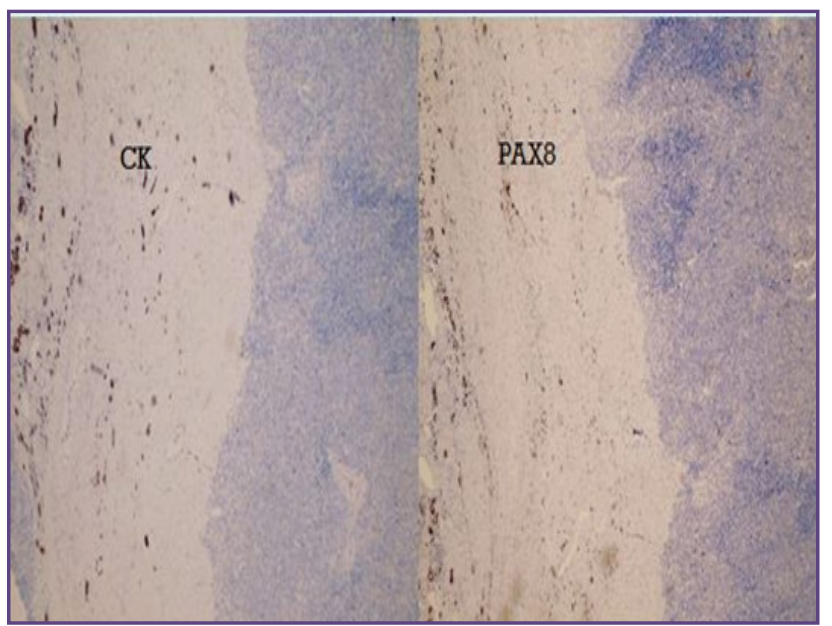

Fig. 4: (IHC stain 20x) Clear cell sarcoma of kidneyCK \& Pax8 negative in tumor cells.

pediatric renal tumors ${ }^{[11]} \& 85 \%$ in another study done by Roy et al ${ }^{[12]}$.

Advanced clear cell renal cell carcinoma is highly resistant to cytotoxic chemotherapy drugs. Thus differentiation of CCSK from clear cell renal cell carcinoma of kidney and undifferentiated adult renal neoplasm including sarcomatoid renal cell carcinoma is especially important in adult patients. ${ }^{[2]}$ Prognosis of CCSK depends upon stage, age at diagnosis \& tumor necrosis. Stage IV \& young age are adverse prognostic factors for event free survival.

Total Radical nephrectomy followed by postoperative chemotherapy and flank radiation therapy is the treatment of choice of CCSK. The tumor is highly responsive to combination treatment with doxorubicin, vincristine and 
actinomycin. The role of doxorubicin in therapy emphasizes the need for pathologists to accurately identify CCSK ${ }^{[1]}$.

This case report highlights the importance in making accurate diagnosis in adult patients with CCSK suspected clear cell renal cell carcinoma. In particular, appropriate selection of IHC ensures timely diagnosis and improves patient management.

\section{Conclusion}

We concluded that clear cell sarcoma of kidney in adult is rare. Unfamiliar morphological patterns can cause difficulty in diagnosis. Awareness about its morphological patterns and diligent use of Immunohistochemistry will help in their identification. This is important as most of them can be cured and have good survival rate by adequate excision followed by chemo-radiotherapy. Its differentiation with clear cell renal cell carcinoma is necessary which is highly resistant to traditional cytotoxic chemotherapy.

\section{Conflict of Interest}

The authors declare that there is no conflict of interests regarding the publication of this paper.

\section{Refernces}

1. Argani.P, Perlman EJ, Breslow NE, Browning, Green DM, D" Angio GJ, Beckwith JB, Clear cell sarcoma of the kidney: a review of 351 cases from the National Wilms Tumor Study Group Pathology Center. Am j Surg Pathol 2000; 24: 4-18.

2. Amin MB, de Peralta-Venturina MN, Ro JY, El- Naggar A, Mackay B, Ordonez N. Clear cell sarcoma of kidney in an adolescent and in young adults; a report of four cases with ultrastructural, immunohistochemical, and DNA flow cytometric analysis. Am J Surg Pathol. 1999 Dec; 23(12):1455- 63 .
3. Adanani A, Lalid R, Bouklata S, Ajana A, Hammani L, Imani F. Clear cell sarcoma of kidney in an adult: a case report. J Radiol. 2006; 87:136-8.

4. Benchekroun A, Zannoud M, el Alj HA, Nouini Y, Marzouk M, Faik M. Clear cell sarcoma of the kidney: three case reports. Prog Urol. 2002; 12(3):469-73.

5. Lanka K, Bhramaramba K, Venkata Ramana P, Sunethri P. Clear cell sarcoma of kidney in an adult: an extremely rare case. Int Surg J 2015; 2:289-91.

6. Beckwith JB. Wilms' tumor and other renal tumors of childhood: a selective review from the National Wilms' Tumor Study Pathology Center. Hum Pathol. 1983; 14:48192.

7. Morgan E, Kidd JM. Undifferentiated sarcoma of the kidney: a tumor of childhood with histopathologic and clinical characteristics distinct from Wilm's tumor. Cancer 1978; 42:1916-21.

8. Marsden HB, Lawler W. Bone metastasizing renal tumour of childhood: histopathological and clinical review of 38 cases. Vir- chows Arch [A] 1980; 387:341-51.

9. Mirkovic J, Calicchio M, Fletcher C D \& Perez-AtaydeR . Diffuse and strong cyclin D1 immunoreactivity in clear cell sarcoma of the kidney, Histopathology 2015; 67: 306-12.

10. Haas JE, Bonadio JF, Beckwith JB. Clear Cell Sarcoma of the Kidney with emphasis on ultrastructural studies. Cancer 1984; 54:2978-2987.

11. Ueno-Yokohata $\mathrm{H}$. et al. Consistent in-frame internal tandem duplications of BCOR characterize clear cell sarcoma of the kidney. Nat. Genet. 2015; 47: 861-63.

12. Roy, A., Kumar, V., Zorman, B., Fang, E., Haines, K. M., Doddapaneni, H., Parsons, D. W. Recurrent internal tandem duplications of BCOR in clear cell sarcoma of the kidney. Nature Communications. 2015; 6: 8891.

*Corresponding author:

Dr Pragya Nirav Vakani, Nirmaan Pathology Laboratory 101, Ashoka Complex, Sardar Patel Stadium Road, Navrangpura, Ahmedabad,

Gujarat. India. 380014

Email: drpragya23@gmail.com

Financial or other Competing Interests: None. 